\title{
Endoscopic management of retained patency capsules
}

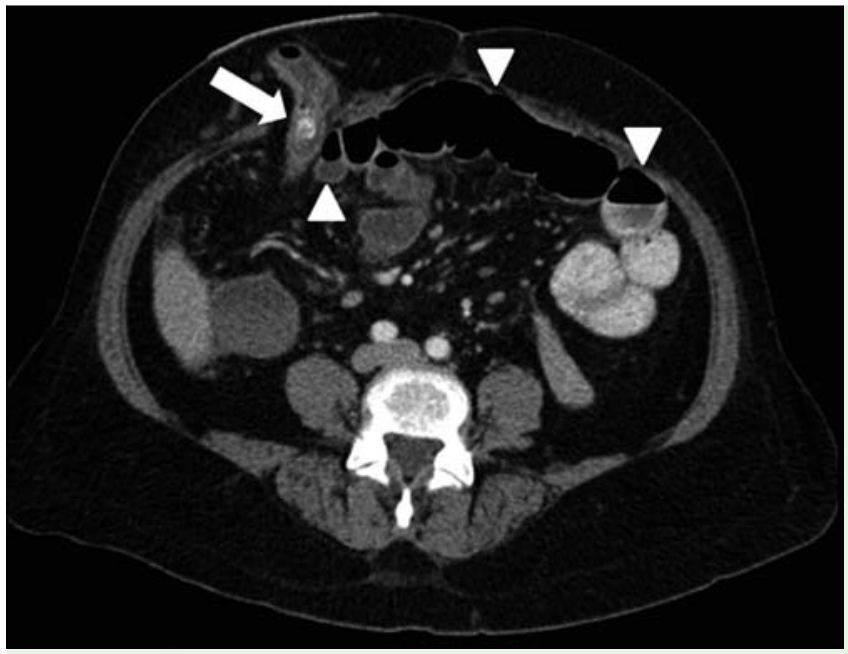

Fig. 1 Computed tomography (CT) scan of the abdomen of a 58-year-old woman with pain at her ileostomy site for the previous 5-6 hours. She had ingested a patency capsule earlier that day. The patency capsule can be seen (arrow) $3-5 \mathrm{~cm}$ proximal to the ileostomy opening and dilated proximal loops of bowel with air-fluid levels (arrowheads).

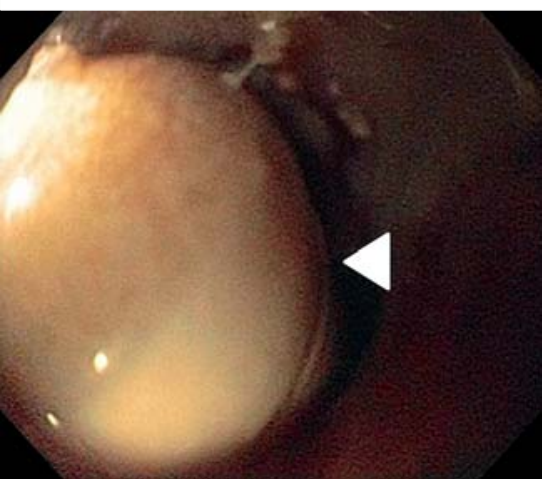

Fig.3 Intact patency capsule (arrowhead) seen proximal to the ileal stricture.

A 58-year-old woman presented to the emergency room with pain at her ileostomy site for the previous 5-6 hours. She had had a colonic resection for "ulcerative colitis" 15 years earlier. A computed tomography (CT) scan of the abdomen demonstrated a foreign body in the ileostomy opening with proximal small-bowel obstruction ( Fig. 1). She had ingested a patency capsule earlier that day before capsule enteroscopy for ongoing work-up of iron deficiency anemia. Ileoscopy showed a stricture in the terminal ileum ( Fig.2), which was dilated with 8-12-Fr through-the-scope balloons. An intact patency capsule proximal to the stricture was retrieved with a Roth Net retrieval device ( Fig.3). Ileitis

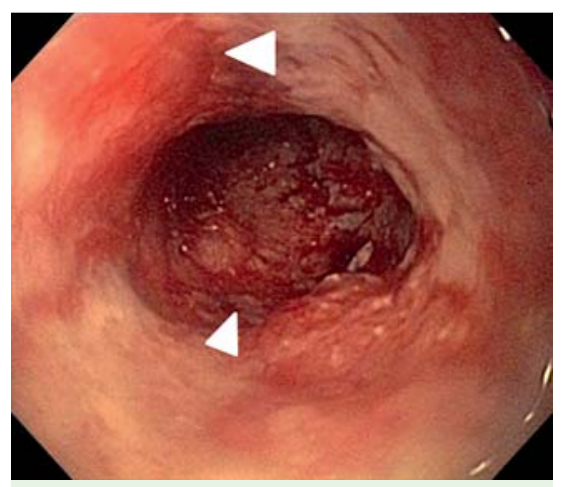

Fig.4 lleitis (arrowheads) seen proximal to the ileal stricture after removal of the patency capsule.

was noted up to $15-20 \mathrm{~cm}$ cephalad from the ileostomy opening ( Fig.4). Active inflammation with granulation tissue was seen in ileal biopsies suggesting an inflammatory stricture.

A 67-year-old man was undergoing outpatient work-up for iron deficiency anemia. Prior esophagogastroduodenoscopy and colonoscopy findings were unremarkable. He reported chronic ibuprofen use for musculoskeletal pain. A patency capsule was administered before capsule enteroscopy because of a history of partial small-bowel obstruction 2 weeks earlier. The capsule was seen in the right lower quadrant on X-ray 2 days later. The patient was completely asymptomatic. A device-assisted lower enteroscopy per-

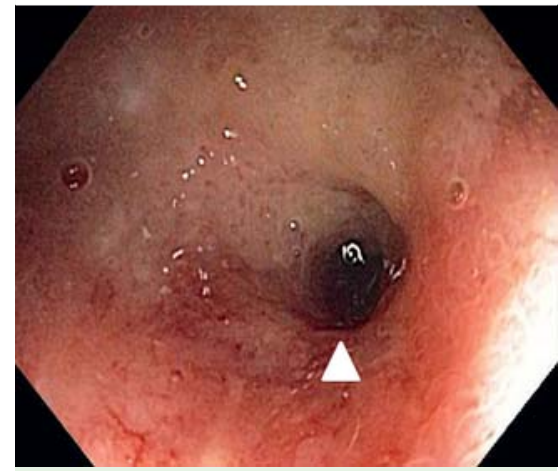

Fig. 2 lleoscopy showing a stricture in the distal ileum (arrowhead).

formed 2 weeks later for his iron deficiency anemia showed unremarkable findings up to $18 \mathrm{~cm}$ distal from the ileum. A device-assisted upper double-balloon enteroscopy 4 weeks later showed an intact patency capsule proximal to a $2-\mathrm{cm}$, cratered ulcer in the distal ileum with stricture formation. The stricture was dilated with 8-12-Fr through-the-scope balloons. The patency capsule passed through the dilated stricture. The stricture biopsy showed acute on chronic inflammation with granulation tissue formation. The iron deficiency anemia and stricture were attributed to chronic nonsteroidal anti-inflammatory drug use. In conclusion, patency capsules may be retained in patients with unknown strictures. Prompt endoscopic intervention in symptomatic patients may avoid surgical intervention.

Endoscopy_UCTN_Code_CPL_1AI_2AB

\section{Competing interests: None}

\section{Shashank Garg1', Rohit Anand', Ethan Dubin², Sergey Kantsevoy ${ }^{3}$, Sudhir Dutta ${ }^{2,4}$}

${ }^{1}$ Johns Hopkins University-Sinai Hospital Program in Internal Medicine, Department of Medicine, Sinai Hospital, Baltimore, Maryland, USA

2 Division of Gastroenterology, Department of Medicine, Sinai Hospital, Baltimore, Maryland, USA 
${ }^{3}$ Institute for Digestive Health \& Liver Disease, Department of Medicine, Mercy Medical Center, Baltimore, Maryland, USA

${ }^{4}$ Department of Medicine, University of Maryland School of Medicine, Baltimore, Maryland, USA

\section{Bibliography}

Dol http://dx.doi.org/

10.1055/s-0034-1390839

Endoscopy 2014; 46: E662-E663

(c) Georg Thieme Verlag KG

Stuttgart · New York

ISSN 0013-726X
Corresponding author

Shashank Garg

1881 Star Shoot Parkway

Lexington

KY 40509

USA

Fax: +1-859-257-4937

shashgarg87@gmail.com 